\title{
AB INITIO DETERMINATION OF BAND STRUCTURES OF VIBRATIONAL SPECTRA OF NON-RIGID MOLECULES. APPLICATIONS TO METHYLAMINE AND DIMETHYLAMINE.
}

\author{
Y.G. Smeyers, M.L. Senent and M. Villa ${ }^{1}$ \\ Instituto de Estructura de la Materia, C.S.I.C. \\ calle Serrano, $n^{\circ} 123$, E-28006 MADRID
}

\begin{abstract}
A bstract
The ab initio determination of the band structures of infrared spectra is described giving as examples the methyl torsion and amine wagging in methylamine and the double torsion in dimethylamine. In addition, the influence of the amine hy. drogen symmetric bending and the $\mathrm{CNC}$ skeleton symmetric bending is considered in methylamine and dimethylamine, respectively. For this purpose, the potentia. energy sufaces and kinetic parameters are determined at the RHF/MP2 levels with large basis sets. The numerical results are fitted as a function of the vibrational angles to conveniently symmetry adapted functional forms. The Schrödinger equations for the nuclear motions solved by expanding the solutions into products of trigonometric functions. From the energy levels, the vibrational functions and the electric dipole moment variations the bands locations and intensities are determined. The calculated spectra are compared with the available experimental data. In the case of methylamine, the torsional splittings and frequencies are relatively well reproduced, whereas the wagging frequencies appear to be slightly too high. In the case, of dimethylamine, the symmetric and antisymmmetric torsion modes are very well reproduced, whereas the $\mathrm{CNC}$ bending frequencies appear also to be too high.
\end{abstract}

\section{List of Contents}

1. Introduction

2. Theory

3. Applications

3.1. Methyl Torsion and Amine Hydrogen Wagging in Methylamine

3.2. Amine Hydrogen Wagging and Bending in Methylamine

3.3. Double Torsion in Dimethylamine

3.4. Double Torsion + Bending in Dimethylamine

4. Conclusions

\footnotetext{
${ }^{1}$ Permanent Address: Departamento de Química, UAM, Iztapalapa, Av.Purisina y Michoacan CP 09340, MEXICO D.F.

ADVANCES IN QUANTUM CHEMISTRY. VOLUME 32 


\section{INTRODUCTION}

A non-rigid molecule is defined as a molecular system possessing large amplitude vibrational modes. These large amplitude motions occur when the molecule is flexible and especially when it possesses various isoenergetic conformations separated by relatively low energy barriers. These motions use to be internal rotations or inversions which give rise to large progressions in the electronic, far infrared (FIR) and Raman spectra [1-3].

In the following, the calculations of the methylamine and dimethylamine far infrared spectra will be considered. Methylamine will be considered in two different ways: the methyl torsion and the amine hydrogen atom wagging; and the amine hydrogen wagging, and symmetric bending discarding the methyl torsion, in order to study the influence of the bending on the wagging mode. Dimethylamine will be considered also in two different ways: the double methyl rotation, and the double methyl rotation plus the CNC angle symmetric bending.

In this aim, the technique developed for determining the acetone far infrared spectrum [4] is adopted. In this, the potential energy surface on which the nuclei are moving is determined, the nuclear motion Schrödinger equation is solved, and from the energy levels, torsional functions and the dielectric moment variations the FIR spectrum is built up.

\section{THEORY}

The potential energy surface on which the nuclei are moving is determined assuming the Born-Oppenheimer approximation as well as the separability of the small and large amplitude motions. It is not always easy to distinguish between large and not so large amplitude motions. One criterion can be the energy levels. Usually, motions between isoenergetic conformations as well as vibrational motions affected seriously by the former are considered together.

Under these conditions, the molecule is supposed to be in equilibrium with respect to the small amplitude vibration modes. As a result, the total energy of each conformation of the non-rigid coordinate space is determined by a reliable ab initio procedure with full optimization of the geometry with respect to the other coordinates.

From the optimized geometry the kinetic parameters are deduced, for each conformation, by inversion of the inertial matrix according to the Harthcock and Laane's procedure [5]: 


$$
\left(\begin{array}{cc}
I & X \\
X^{t} & Y
\end{array}\right)
$$

where $I$ is the inertial tensor corresponding to the overall rotation $Y$ is the vibrational submatrix (here diagonal), and $X$ is the interaction matrix between the external and internal motions.

In particular we have:

$$
\begin{gathered}
X_{i x}=\sum_{a} m_{a}\left(\vec{r}_{a} \times \frac{\partial \vec{r}_{a}}{\partial \alpha_{i}}\right)_{x} \\
Y_{i j}=\sum_{a} m_{a}\left(\frac{\partial \vec{r}_{a}}{\partial \alpha_{i}}\right) \bullet\left(\frac{\partial \vec{r}_{a}}{\partial \alpha_{j}}\right)
\end{gathered}
$$

where $m_{a}$ is the mass of atom a, and $\vec{r}_{a}$ its displacement vector.

Finally, the numerical results for the potential and kinetic parameters are adjusted to a symmetry adapted functional form.

In order to solve the Schrödinger equation for the nuclear motions, the vibrational solutions are developed in terms of some basis functions, and the Hamiltonian matrix diagonalized, yielding the energy levels and the wave functions. As basis functions, the symmetry vectors which factorize into boxes the Hamiltonian matrix are conveniently used.

This technique assumes previous knowledge of the symmetry eigenvectors which may be deduced by using Group Theory for Non-Rigid Molecules as well as some information about the necessary basis length $[2,3]$.

In the spectrum calculations the band frequencies and intensities are determined in absolute value without any scale factor. The band frequencies are determined by the difference between the energy levels, of initial and final states. The intensities are determined through the electric dipole moment variation approach [6]:

$$
I_{i f}=\frac{g}{3 B R^{2} e^{2}}\left[\epsilon_{i}-\epsilon_{f}\right]\left[C_{i}-C_{f}\right]<\varphi_{i}|\vec{\mu}| \varphi_{g}>^{2}
$$

where $\epsilon, \mathrm{C}$ and $\varphi$ are the energies, populations and vibrational functions of the initial (i) and final (f) states. $R$ and $B$ are the average rotation radii and kinetic parameters, $\vec{\mu}$ is the dipole moment written as a function of the large amplitude coordinates. $\vec{\mu}$ is obtained in the same calculations for the potential. The populations are given by the Boltzmann statistics and the dipole moment components are conveniently shifted along the principal inertial axes of the molecule.

In order to determine the band profiles, the shape of the molecule has to be considered [7]. As is well known, in symmetric prolate or oblate tops, the 
dipole variation along the main principal axis ( $A$ or $C$ ) gives rise to a or $\mathbf{c}$ type bands, respectively, with a sharp Q branch. On the contrary, the perpendicular dipole moment variation gives rise to $b-c$ or $a-b$ type bands with broad PR branches. Generally, only the sharp bands, which can be more easily compared with the experimental data are considered.

Furthermore, we have to remark that Group Theory for Non-Rigid Molecules may be advantageousiy used to deduce a symmetry adapted analytical form for the potential, as well as the symmetry eigenvectors for simplifying the Hamiltonian matrix solution. In the same way, Group Theory permits to label and classify the energy' levels and the vibrational functions. Finally, it may be also used to deduce selection rules for the infrared transitions.

\section{APPLICATIONS}

\subsection{Methyl Torsion and Amine Hydrogen Wagging in Methylamine}

Let us consider first the methyl internal rotation and the amine hydrogen wagging in methylamine (Fig. 1), taking as origin for the torsion and wagging angles the molecular plane of methylamine in a planar conformation. The Non-Rigid Group for the methyl torsion and amine hydrogen waging in methyl amine has been derived in [8] into the Longuet-Higgins formalism, and in [9] into the physical operations formalism.
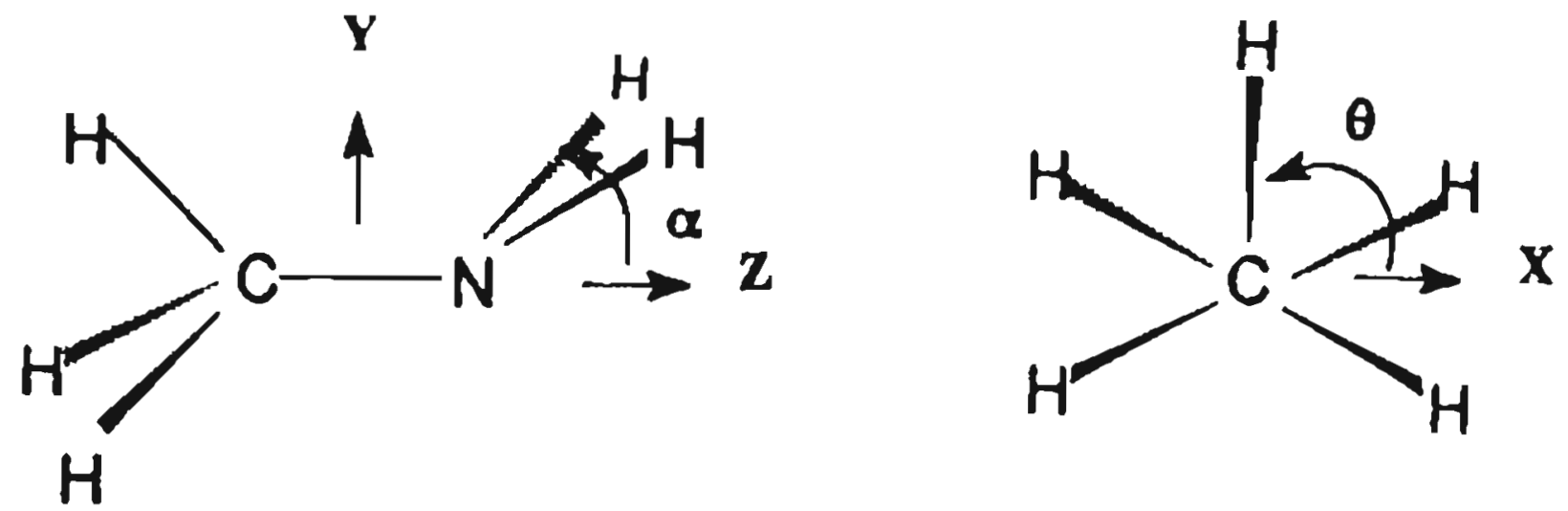

Figure 1. Large amplitude motions in methylamine: torsion $(\theta)$ and wagging $(\alpha)$. 
The Hamiltonian operator restricted to these two motions takes the form:

$$
\begin{gathered}
H(\theta, \alpha)=-\frac{\partial}{\partial \theta} B_{\theta}(\theta, \alpha) \frac{\partial}{\partial \theta}-\frac{\partial}{\partial \theta} B_{\theta, \alpha}(\theta, \alpha) \frac{\partial}{\partial \alpha}-\frac{\partial}{\partial \alpha} B_{\alpha, \theta}(\theta, \alpha) \frac{\partial}{\partial \theta}-\frac{\partial}{\partial \alpha} B_{\alpha}(\theta, \alpha) \frac{\partial}{\partial \alpha} \\
+V(\theta, \alpha)
\end{gathered}
$$

It is easily seen to be invariant under the following operations [9]:

1) A threefold rotation of the methyl group:

$$
\hat{C}_{3} f(\theta, \alpha) \equiv f\left(\theta+\frac{2 \pi}{3}, \alpha\right)
$$

2) A twofold rotation of the methyl group followed by a sign change of the rotation:

$$
\hat{U} f(\theta, \alpha) \equiv f(\pi-\theta, \alpha)
$$

3) A simultaneous sign change of rotation and wagging angles, (or double switch):

$$
\hat{V} f(\theta, \alpha) \equiv f(-\theta,-\alpha)
$$

Notice that the double switch operation exists always when there are symmetry plane in the moving moities and the frame of the molecule [10]. These operations are illustrated in figure 2. With these definitions, the restricted Non-Rigid Group ( $\mathrm{r}-\mathrm{NRG}$ ) for the torsion and wagging mode is a group of order twelve, isomorphic to the $C_{6 v}$ symmetry point group:

$$
C_{3}^{I} \wedge\left[V^{I} \times U^{I}\right]=G_{12} \sim C_{6 v}
$$

The subgroups $C_{3}^{I}, U^{I}$ and $V^{I}$ are defined by the operations (5),(6) and (7), respectively. The character table as well as the symmetry eigenvectors of this group are given in Ref [9].

In particular, we have for the potential energy function, the symmetry adapted analytical form:

$$
V(\theta, \alpha)=\sum_{k=\text { even }, L} A_{K L} \cos 3 K \theta \cos L \alpha+\sum_{k=\text { odd }, L} B_{K L} \sin 3 K \theta \sin L \alpha
$$

where no $\cos \times \sin$ mixed term appears.

The far infrared spectrum of methylamine has bee studied mainly by Onashi and coworkers [11,12], and Kreglewski et al $[13,14]$. 
The potential energy calculations were performed, at the RHF/MP2 level, with the $6-311 \mathrm{G}++(3 \mathrm{fd}, 3 \mathrm{dp})$ basis set, for 36 selected values of the torsion and wagging angles. In this aim, the GAUSSIAN 92 program package was resorted [15]. The following values were used for the torsion, $0^{\circ}, 30^{\circ}, 60^{\circ}$ and $90^{\circ}$, and for the wagging, $-69.0^{\circ},-54.540845^{\circ},-38.0^{\circ},-19.0^{\circ}, 0.0^{\circ}, 19.0^{\circ}, 38.0^{\circ}$, $54.540845^{\circ}$ and $69.0^{\circ}$. The preferred conformations were found at $\theta=0.0^{\circ}$ and $\alpha= \pm 54.540845^{\circ}$ The fit of the relative energy values with respect to the lowest one by a least square method to the equation (9) yields an expression given in Ref.[16].

The $\mathrm{B}_{i}$ kinetic parameters were recalculated for each of the 36 configurations, without the corrections given in Ref. [14,16], and fitted to the same symmetry adapted analytical form (9). The torsion kinetic parameters resemble those of Ref. [16]. In contrast the wagging ones (equilibrium value 26.729 $\left.\mathrm{cm}^{-1}\right)$ differ substantially from those used in [16] $\left(9.602 \mathrm{~cm}^{-1}\right)$, as well as to the approximate experimental value proposed by Onashi et al.[11] $\left(9.19 \mathrm{~cm}^{-1}\right)$.

The torsional-wagging nuclear equation was solved at two levels of approximations: using either the variable kinetic parameters either the equilibrium value. In this aim, $31 \times 41$ trigonometric functions were used for describing the methyl torsion and the amine hydrogen atom wagging, respectively. The energy levels encountered are given in Table 1, where it is seen that the both approaches yield similar results. The levels are clustered into quartets. In fact it is a matter of sextets taking into account the degeneracies.

The first quartet corresponds to the fundamental level. The second and third to the first and second torsionally excited levels. The fourth quartet corresponds to the first wagging excited level. This assignment rests on the intensities given in Table 2. The dipole moment variations due to a wagging transition must be indeed expected to be much larger than those due to a torsional transition.

It is seen that the first excited torsional levels agree fairly well with the value deduced from experiment [14], whereas the wagging level appears to be too low. In particular, the first excited wagging level is found at $830 \mathrm{~cm}^{-1}$ instead of $720 \mathrm{~cm}^{-1}$ [14].

This discrepancy could be attributed to the interactions between the wagging and bending motions of the amine hydrogen atoms. If the bending angle is plotted versus the wagging angle a large dependency is indeed detected. The bending angle exhibits the largest value close to $120^{\circ}$ ( $\mathrm{sp}^{2}$ hybridization) when the amine group is forced to be in the molecular plane. On the contrary, the bending angle presents the smallest values ( $\mathrm{sp}^{3}$ hybridization) when the amine group adopts a pyramidal conformation [16].

The splittings between the torsional fundamental sublevels for constant and 
variable $B_{\theta}$ are seen to be 0.353 and $0.435 \mathrm{~cm}^{-1}$ in relatively good agreement with the experimental value of $0.249 \mathrm{~cm}^{-1}$ given by Onashi et al.[11].

Table 1. Energy levels (in $\mathrm{cm}^{-1}$ ) for the torsion and wagging vibration modes in Methylamine obtained with the constant and variable (B) kinetic parameters.

\begin{tabular}{|c|c|c|c|c|c|c|c|}
\hline \multirow{2}{*}{\multicolumn{2}{|c|}{ Levels }} & \multirow[t]{2}{*}{ Symmetry } & \multirow{2}{*}{$\begin{array}{c}\text { Energy in } \mathrm{cm}^{-1} \\
\mathrm{~B} \text { const. }\end{array}$} & \multicolumn{3}{|c|}{ Vibrational mode } & \multirow[t]{2}{*}{ Exp.[14] } \\
\hline & & & & $B$ varia. & $\nu_{15}$ & $\nu_{9}$ & \\
\hline \multirow{3}{*}{1} & & $A_{1}$ & 609.697 & 602.555 & \multirow{4}{*}{0} & \multirow{4}{*}{0} & \multirow{4}{*}{600} \\
\hline & -1 & $\mathrm{E}_{1}$ & 610.051 & 602.990 & & & \\
\hline & -2 & $\mathrm{~B}_{2}$ & 609.899 & 603.057 & & & \\
\hline 2 & -3 & $\mathrm{E}_{2}$ & 610.142 & 603.243 & & & \\
\hline 3 & -4 & $\mathrm{E}_{2}$ & 864.987 & 859.860 & \multirow{4}{*}{1} & \multirow{4}{*}{0} & \multirow{4}{*}{870} \\
\hline 4 & -5 & $\mathrm{E}_{1}$ & 865.294 & 860.631 & & & \\
\hline \multirow[t]{2}{*}{5} & & $A_{1}$ & 872.070 & 866.709 & & & \\
\hline & -6 & $\mathrm{~B}_{2}$ & 872.750 & 868.414 & & & \\
\hline \multirow[t]{2}{*}{6} & & $A_{1}$ & 1045.610 & 1041.845 & \multirow{4}{*}{2} & \multirow{4}{*}{0} & \multirow{4}{*}{1010} \\
\hline & -7 & $\mathrm{~B}_{2}$ & 1046.093 & 1042.972 & & & \\
\hline 7 & -8 & $\mathrm{E}_{1}$ & 1086.093 & 1083.324 & & & \\
\hline 8 & -9 & $\mathrm{E}_{2}$ & 1086.965 & 1083.921 & & & \\
\hline 9 & -10 & $\mathrm{E}_{2}$ & 1220.800 & 1218.555 & \multirow{4}{*}{3} & \multirow{4}{*}{0} & \multirow{4}{*}{1310} \\
\hline 10 & -11 & $\mathrm{E}_{1}$ & 1220.937 & 1018.804 & & & \\
\hline \multirow[t]{2}{*}{11} & & $\mathrm{~B}_{1}$ & 1363.315 & 1361.840 & & & \\
\hline & -12 & $\mathrm{~A}_{2}$ & 1363.459 & 1362.047 & & & \\
\hline \multirow[t]{2}{*}{12} & & $\mathrm{~B}_{2}$ & 1375.089 & 1373.934 & \multirow[b]{2}{*}{4} & \multirow[b]{2}{*}{0} & \multirow{6}{*}{1315} \\
\hline & -13 & $A_{1}$ & 1376.628 & 1375.643 & & & \\
\hline \multicolumn{2}{|l|}{13} & $A_{1}$ & 1391.518 & 1409.130 & \multirow{4}{*}{0} & \multirow{4}{*}{1} & \\
\hline 14 & -14 & $E_{1}$ & 1395.773 & 1415.389 & & & \\
\hline \multirow[t]{2}{*}{15} & -15 & $E_{2}$ & 1404.130 & 1428.379 & & & \\
\hline & -16 & $B_{2}$ & 1409.621 & 1436.863 & & & \\
\hline 16 & -17 & $\mathrm{E}_{2}$ & 1558.542 & 1558.314 & 4 & 0 & \\
\hline 17 & -18 & $\mathrm{E}_{1}$ & 1559.592 & 1559.464 & & & \\
\hline 18 & & $\mathrm{~B}_{1}$ & 1608.333 & 1627.357 & & & \\
\hline 19 & -19 & $\mathrm{E}_{2}$ & 1607.474 & 1627.357 & 1 & 1 & \\
\hline 20 & -20 & $\mathrm{E}_{1}$ & 1625.599 & 1658.956 & & & \\
\hline & -21 & $\mathrm{~A}_{2}$ & 1657.308 & 1699.178 & & & \\
\hline
\end{tabular}


In Table 2, the calculated frequencies and intensities are given. Unfortunately, the FIR spectrum of methylamine is too crowded to permit a reliable comparison. The first torsional frequency $257.3 \mathrm{~cm}^{-1}$, however, appears to be in very good agreement with the experimental value $262.7 \mathrm{~cm}^{-1}$ [11].

Table 2. Far infrared frequencies (in $\mathrm{cm}^{-1}$ ) and relative intensities for the $\mathbf{c}$ type torsional and wagging bands in methylamine obtained from ab initio calculations with the variable kinetic parameters.

\begin{tabular}{|lcccc|}
\hline $\begin{array}{l}\text { Assignments } \\
\nu \nu^{\prime} \rightarrow \nu \nu^{\prime}\end{array}$ & Symmetries & Frequencies & Intensities \\
\hline 00 & 10 & & & \\
& & $E_{1} \rightarrow E_{2}$ & 256.87 & $1.10^{-4}$ \\
& $E_{2} \rightarrow E_{1}$ & 257.39 & $1.10^{-4}$ \\
\hline 10 & 20 & & & \\
& & $E_{1} \rightarrow E_{2}$ & 221.67 & - \\
& $E_{2} \rightarrow E_{1}$ & 221.71 & - \\
\hline 00 & 01 & & & \\
& & $B_{2} \rightarrow A_{1}$ & 806.07 & 0.0858 \\
& & $E_{2} \rightarrow E_{1}$ & 812.15 & 0.0531 \\
& $E_{1} \rightarrow E_{2}$ & 825.39 & 0.0543 \\
& $A_{1} \rightarrow B_{2}$ & 834.31 & 0.0837 \\
\hline 10 & 11 & & & \\
& & $A_{2} \rightarrow B_{1}$ & 758.94 & 0.0213 \\
& & $E_{2} \rightarrow E_{1}$ & 771.68 & 0.0206 \\
& $E_{1} \rightarrow E_{2}$ & 799.10 & 0.0197 \\
& $B_{1} \rightarrow A_{2}$ & 832.47 & 0.0206 \\
\hline
\end{tabular}

\subsection{Amine Hydrogen Wagging and Bending in Methylamine}

In order to verify the interaction between the amine hydrogen atoms bending and wagging modes, the energy levels corresponding to these motions are calculated discarding the torsion. The three-dimensional problem will be considered in a next future. For this purpose, let us take as before as origin for the wagging angle the molecular plane of methylamine in a planar conformation. The bending coordinate is the $\mathrm{H}-\mathrm{N}-\mathrm{H}$ angle.

The Hamiltonian operator restricted to these two motions is similar to the previous one (4) in which the $\theta$ variable has to be replaced by the $\beta$ one. This operator is easily seen to be invariant under the following dynamical operations: 
1) A change of sign of the wagging angle:

$$
\hat{U}_{\alpha} f(\alpha, \beta)=f(-\alpha, \beta)
$$

2) A change of sign of the bending angle:

$$
\hat{U}_{\beta} f(\alpha, \beta)=f(\alpha,-\beta)
$$

Operation 1) is closely related with the full optimization of the molecular geometry. In particular, the conformation of the methyl group adopts the energy minimum for any values of $\alpha$ and $\beta$, in such a way that it may be considered as planar.

As a result, the $\mathrm{r}-\mathrm{NRG}$ for these motions may be written as:

$$
\left[U_{\alpha}^{I} \times U_{\beta}^{I}\right]=G_{4} \sim C_{2 v}
$$

which is a group of order four isomorphic with the symmetry point group $C_{2 v}$, with the irreducible representations $A_{1}, A_{2}, B_{1}$ and $B_{2}$.

In this expression, the subgroups $U_{\alpha}^{I}$ and $U_{\beta}^{I}$ are defined from the operations (10) and (11), respectively.

In these conditions, the potential energy function for these two motions takes the symmetry adapted analytical form:

$$
V(\alpha, \beta)=\sum_{K} \sum_{L} \cos (K \alpha) \cos (L \beta)
$$

The energy of methylamine was determined for a set of 49 wagging and bending angles using the same approach: RHF/MP2 with $6-311 \mathrm{G}++(3 \mathrm{df}, 3 \mathrm{dp})$ basis set, and with full optimization of the geometry with respect to the other coordinates including the torsion. For the bending the following values were considered: $91.27 .30^{\circ}, 96.2730^{\circ}, 101.2730^{\circ}, 106.2730^{\circ}, 111.2730^{\circ}, 116.2730^{\circ}$ and $121.27 .30^{\circ}$, i.e., each $5.0^{\circ}$ from $-15.0^{\circ}$ to $15.0^{\circ}$ arround the minimum found at $106.2730^{\circ}$. For each of these bending values a different optimum wagging angle was found. This was included in the set of wagging angles used in the calculations: $0.0^{\circ}, 15.0^{\circ}, 30.0^{\circ}, 45.0^{\circ}, 60.0^{\circ}$, and $70.0^{\circ}$.

These electronic energy values were fitted to expression (13), as well as the kinetic parameters obtained from the optimum geometries. For solving the Schrödinger equation, the solutions were developed on $41 \times 41$ trigonometric functions for the wagging and bending, respectively. The energy levels are given in Table 3. 
Table 3. Energy levels (in $\mathrm{cm}^{-3}$ ) for the wagging and bending vibration modes in Methylamine.

\begin{tabular}{|rcccc|}
\hline Levels & Symmetry & Energy in $\mathrm{cm}^{-1}$ & \multicolumn{2}{c|}{ Vibrational mode } \\
& & & $\nu_{9}$ & $\nu_{4}$ \\
\hline 0 & $\mathrm{~A}_{1}$ & 1230.34 & & 0 \\
-1 & $\mathrm{~B}_{1}$ & 1230.34 & 0 & \\
-2 & $\mathrm{~B}_{2}$ & 1231.20 & & \\
1 & $\mathrm{~A}_{2}$ & 1231.20 & & \\
& & & & \\
-3 & $\mathrm{~B}_{1}$ & 1969.86 & & \\
2 & $\mathrm{~A}_{1}$ & 1969.86 & 1 & \\
3 & $\mathrm{~A}_{2}$ & 2000.25 & & \\
-4 & $\mathrm{~B}_{2}$ & 2000.25 & & \\
& & & & \\
4 & $\mathrm{~A}_{1}$ & 2524.90 & & \\
-5 & $\mathrm{~B}_{1}$ & 2524.90 & 2 & \\
-6 & $\mathrm{~B}_{2}$ & 2724.62 & & \\
5 & $\mathrm{~A}_{2}$ & 2724.62 & & \\
& & & & \\
-7 & $\mathrm{~B}_{1}$ & 2859.01 & & \\
6 & $\mathrm{~A}_{1}$ & 2859.01 & 0 & 1 \\
7 & $\mathrm{~A}_{2}$ & 2881.62 & & \\
-8 & $\mathrm{~B}_{2}$ & 2881.63 & & \\
\hline
\end{tabular}

In this table, it is seen that the levels appear clustered into quartets as to be expected from a four well potential energy surface. The first quartet corresponds to the fundamental level. The first and second excited quartets may be attributed to the wagging mode, whereas the third one to the bending mode. This assignment rests on the theoretical intensities given in Table 4. No strong intensities were obtained between the first and third levels, nor between the second and the fourth ones. In addition, the two wagging transitions present a quasi harmonic behavior. 
Table 4. Far infrared frequencies (in $\mathrm{cm}^{-1}$ ) and relative intensities for the $\mathrm{c}$ type wagging and bending bands in methylamine obtained from ab initio calculations.

\begin{tabular}{|lcccc|}
\hline $\begin{array}{l}\text { Assignments } \\
\nu \nu^{\prime} \rightarrow \nu \nu^{\prime}\end{array}$ & Symmetries & Frequencies & Intensities \\
\hline 00 & 10 & & & \\
& & $A_{1} \rightarrow B_{2}$ & 769.91 & 0.2398 \\
& & $B_{1} \rightarrow A_{2}$ & 769.91 & 0.2397 \\
& & $B_{2} \rightarrow A_{1}$ & 738.67 & 0.2589 \\
& & $A_{2} \rightarrow B_{1}$ & 738.67 & 0.2589 \\
\hline 10 & 20 & & & \\
& & $A_{1} \rightarrow B_{2}$ & 754.76 & 0.0053 \\
& & $B_{1} \rightarrow A_{2}$ & 754.76 & 0.0053 \\
& & $A_{2} \rightarrow B_{1}$ & 524.65 & 0.0134 \\
& $B_{2} \rightarrow A_{1}$ & 524.65 & 0.0134 \\
\hline 00 & 01 & & & \\
& & $B_{1} \rightarrow A_{2}$ & 1651.29 & 0.0255 \\
& $A_{1} \rightarrow B_{2}$ & 1651.29 & 0.0255 \\
& $B_{2} \rightarrow A_{1}$ & 1627.81 & 0.0370 \\
& $A_{2} \rightarrow B_{1}$ & 1627.81 & 0.0370 \\
\hline
\end{tabular}

It is seen that the first excited level is found at $770 \mathrm{~cm}^{-1}$ above the fundamental one in better agreement, but still slightly too high when compared with the experimental value $720 \mathrm{~cm}^{-1}$ [14].

The splitting between the wagging fundamental sublevels is $0.756 \mathrm{~cm}^{-1}$ in some agreement with an hypothetical experimental value of $0.206 \mathrm{~cm}^{-1}$ given by Honashi et al. [11\}.

\subsection{Double Torsion in Dimethylamine}

Let now consider the double methyl torsion in dimethylamine, let us remark that the two methyl groups are superimposable by reflection, see Fig. 2. The Hamiltonian operator is then seen to be invariant under the following dynamical operations [10]:

1) A threefold rotation of each of the methyl group:

$$
\hat{C}_{3} f(\theta)=f\left(\theta+\frac{2 \pi}{3}\right)
$$

2) An exchange-double switch of the two rotation angles: 


$$
\hat{W} \hat{V} f\left(\theta_{1}, \theta_{2}\right)=f\left(-\theta_{2},-\theta_{1}\right)
$$

With such a definition, the r-NRG for the double internal rotation in dimethylamine is a group of order 18 :

$$
\left[C_{3}^{I} \times C_{3}^{\prime I}\right] \times[W V]^{I}=G_{18}
$$

The character table of such a group is given in Refs. $[17,18]$.

In particular, the following symmetry adapted expression is found for the totally symmetric potential energy function:

$$
\begin{gathered}
V\left(\theta_{1}, \theta_{2}\right)=\sum_{K, L=0} A_{K L}\left[\cos 3 K \theta_{1} \cos 3 L \theta_{2}+\cos 3 L \theta_{1} \cos \theta 3 K \theta_{2}\right]+ \\
\sum_{K, L=1} B_{K L}\left[\sin 3 K \theta_{1} \sin 3 L \theta_{2}+\sin 3 L \theta_{1} \cos \theta 3 K \theta_{2}\right]+ \\
\sum_{K=0, L=1} C_{K L}\left[\cos 3 K \theta_{1} \sin 3 L \theta_{2}-\sin 3 L \theta_{1} \cos \theta 3 K \theta_{2}\right]
\end{gathered}
$$

Let us remark that when the third set of terms of this equation is neglected this expression coincides with that for planar acetone of $G_{36}$ symmetry [4]. Since the coefficients of these terms are generally small, both descriptions are considered in the following.

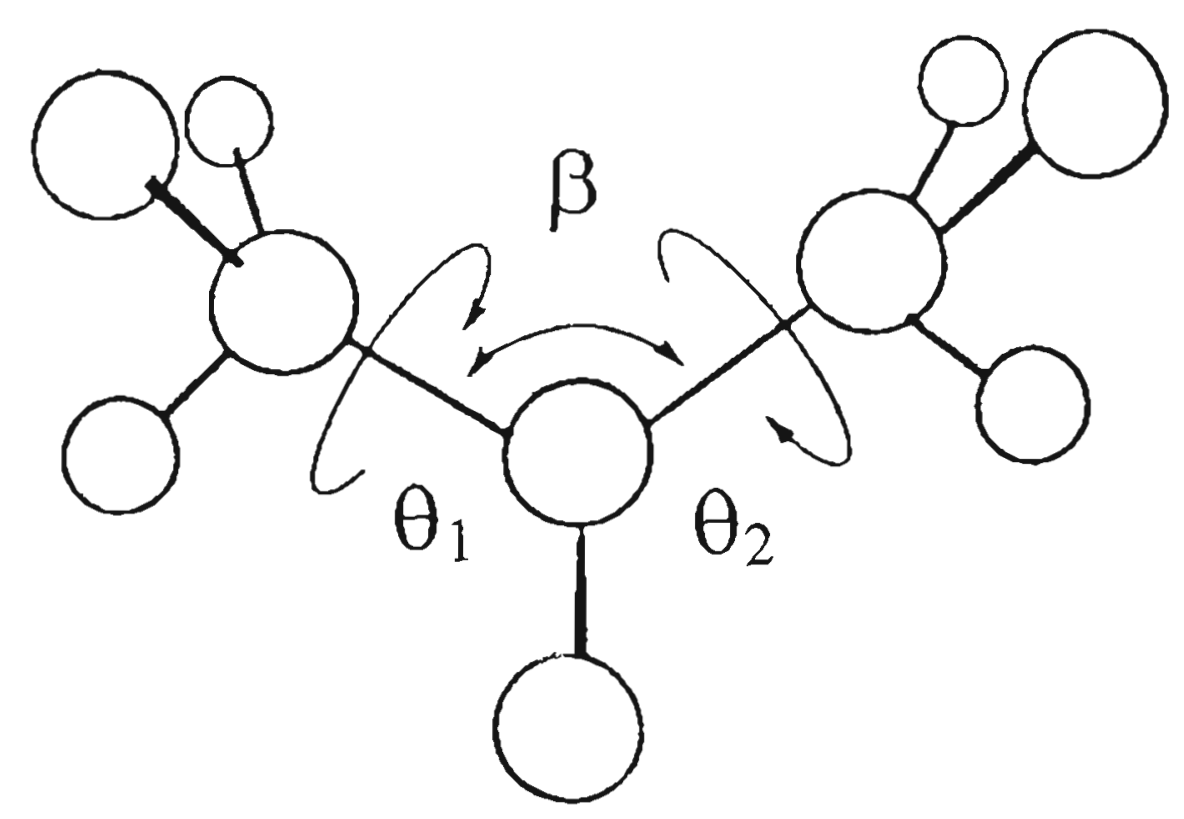

Figure 2. Structure of dimethylamine. The $\theta_{1}$ and $\theta_{2}$, torsional angles, and the $\beta$ bending angle. 
The electronic energy of dimethylamine was determined for ten conformations at the RHF/MP2 level with the $6-311 \mathrm{G}(\mathrm{df}, \mathrm{p})$ basis set. The symmetry criterion was applied to select the conformations for the torsional coordinates. The conformation, in which one of the hydrogen of each methyl group lies on the $\mathrm{CNC}$ molecular plane and pointing outwards, was taken as the origin for the rotations. In this condition, the selected geometries were $\left(0^{\circ}, 0^{\circ}\right),\left(60^{\circ}, 0^{\circ}\right)$, $\left(60^{\circ}, 60^{\circ}\right),\left(30^{\circ}, 0^{\circ}\right),\left(0^{\circ}, 30^{\circ}\right),\left(60^{\circ}, 30^{\circ}\right)\left(30^{\circ}, 30^{\circ}\right),\left(30^{\circ},-30^{\circ}\right)$ and $\left(-30^{\circ}, 30^{\circ}\right)$.

In the most stable conformation, the methyl hydrogen atoms do no lie anymore in the $\mathrm{CNC}$ plane but at $3.3^{\circ}$ and $-3.3^{\circ}$. The wagging and bending angles were found to be equal to $55^{\circ}$ and $111.6^{\circ}$, respectively.

The relative energy values with respect to the most stable conformation were fitted to expression (17) taking the energy minimum as origin for the potential. The kinetic parameters were determined from the optimized geometries by using equation (1), and fitted to the same expression (17).

For solving the Schrödinger equation, the solutions were developed on 37 $\times 37$ trigonometric functions for each torsion mode. In Table 5, the energy levels calculated into the two models, $G_{18}$ and $G_{36}$, are given.

In this table it is seen that the energy levels are clustered into quartets. In fact it is a matter of a ninefold multiplicity taking into account the degeneracies of the $\mathrm{G}$ and $\mathrm{E}$ representations in accordance to the nine potential energy wells. The $G_{36}$ model furnishes sligthly too high levels.

The first quartet corresponds to the fundamental level. The second and fourth to antisymmetric torsionally excited levels. The third, fifth and the last to symmetric torsionally excited levels. The antisymmetric torsion modes correspond to a gearing rotation along the principal diagonal of the potential energy surface. They are active in Raman and somewhat in infrared spectra. The symmetric modes correspond to an antigearing rotation along the secondary diagonal and are active in infrared. 
Table 5. Dimethylamine energy levels $\left(\right.$ in $\mathrm{cm}^{-1}$ )

\begin{tabular}{|c|c|c|c|c|c|}
\hline & & & $V_{3}\left(G_{36}\right)$ & & $V_{3}\left(G_{18}\right)$ \\
\hline \multirow[t]{4}{*}{0} & 0 & $A_{1}$ & 240.392 & $\overline{A_{1}}$ & 237.541 \\
\hline & & $\mathrm{G}$ & 240.392 & $G$ & 237.541 \\
\hline & & $E_{1}$ & 240.392 & $E_{1}$ & 237.541 \\
\hline & & $E_{3}$ & 240.392 & $E_{3}$ & 237.541 \\
\hline \multirow[t]{4}{*}{1} & 0 & $A_{3}$ & 461.562 & $A_{2}$ & 458.767 \\
\hline & & $\mathrm{G}$ & 461.561 & $G$ & 458.766 \\
\hline & & $E_{2}$ & 461.560 & $E_{2}$ & 458.765 \\
\hline & & $E_{3}$ & 461.560 & $E_{3}$ & 458.765 \\
\hline \multirow[t]{4}{*}{0} & 1 & $A_{2}$ & 491.478 & $A_{1}$ & 490.330 \\
\hline & & $G$ & 491.477 & $G$ & 490.329 \\
\hline & & $E_{1}$ & 491.476 & $E_{1}$ & 490.328 \\
\hline & & $E_{4}$ & 491.474 & $E_{3}$ & 490.328 \\
\hline \multirow[t]{4}{*}{2} & 0 & $A_{1}$ & 677.741 & $A_{1}$ & 675.153 \\
\hline & & $G$ & 677.759 & $\mathrm{G}$ & 675.170 \\
\hline & & $E_{1}$ & 677.778 & $E_{1}$ & 675.188 \\
\hline & & $E_{3}$ & 677.778 & $E_{3}$ & 675.188 \\
\hline \multirow[t]{4}{*}{1} & 1 & $A_{1}$ & 701.276 & $A_{2}$ & 700.143 \\
\hline & & $\mathrm{G}$ & 701.304 & $\mathrm{G}$ & 700.171 \\
\hline & & $E_{2}$ & 701.332 & $E_{2}$ & 700.198 \\
\hline & & $E_{4}$ & 701.332 & $E_{3}$ & 700.198 \\
\hline \multirow[t]{4}{*}{0} & 2 & $A_{1}$ & 739.400 & $A_{1}$ & 738.909 \\
\hline & & $\mathrm{G}$ & 739.409 & $\mathrm{G}$ & 738.918 \\
\hline & & $E_{1}$ & 739.418 & $E_{1}$ & 738.927 \\
\hline & & $E_{3}$ & 739.418 & $E_{3}$ & 738.927 \\
\hline \multirow[t]{4}{*}{3} & 0 & $A_{1}$ & 887.090 & $A_{2}$ & 884.838 \\
\hline & & $\mathrm{G}$ & 886.907 & $\mathrm{G}$ & 884.663 \\
\hline & & $E_{2}$ & 886.729 & $E_{2}$ & 884.493 \\
\hline & & $E_{3}$ & 886.729 & $E_{3}$ & 884.493 \\
\hline \multirow[t]{4}{*}{0} & 3 & $A_{2}$ & 982.308 & $A_{1}$ & 982.045 \\
\hline & & $\mathrm{G}$ & 982.287 & $\mathrm{G}$ & 982.024 \\
\hline & & $E_{1}$ & 982.266 & $E_{1}$ & 982.003 \\
\hline & & $E_{4}$ & 982.266 & $E_{3}$ & 982.003 \\
\hline
\end{tabular}


In Tables $6 \mathrm{a}$ and $6 \mathrm{~b}$, the frequencies and intensities are given for the two torsional modes toghether with the experimental data. It is a matter of aand $\mathbf{b}-\mathbf{c}$ hybrid type bands with sharp $Q$ branches. A very good agreement is observed [19]. The relative position of the $\nu_{12}$ and $\nu_{24}$ fundamental frequencies, however, shows a small error: $31.6 \mathrm{~cm}^{-1}$ instead of $36.9 \mathrm{~cm}^{-1}$.

Table 6a. Calculated frequencies (in $\mathrm{cm}^{-1}$ ) and relative intensities for the symmetric $\nu_{12}$ torsion mode (double antigearing rotation) in dimethylamine.

\begin{tabular}{|llllll|}
\hline assign. & freq. & int. & exp. $^{a}[19]$ \\
\hline $0 \rightarrow 0$ & 1 & & & & \\
$A_{1}-A_{1}$ & 252.789 & 3.690 & & \\
$\mathrm{G}-\mathrm{G}$ & 252.788 & 7.379 & 256.3 & vs \\
$E_{1}-E_{1}$ & 252.787 & 1.845 & & \\
$E_{3}-E_{3}$ & & 252.787 & 1.845 & & \\
& & & & & \\
$0 \quad 1 \rightarrow 0$ & 2 & & & & \\
$A_{1}-A_{1}$ & 248.579 & 2.038 & & \\
$\mathrm{G}-\mathrm{G}$ & 248.589 & 4.077 & 250.8 & $\mathrm{~s}$ \\
$E_{1}-E_{1}$ & 248.599 & 1.019 & & \\
$E_{3}-E_{3}$ & 248.599 & 1.019 & & \\
& & & & \\
$0 \quad 2 \rightarrow 0$ & 3 & & & & \\
$A_{1}-A_{1}$ & 243.136 & 0.834 & & \\
$\mathrm{G}-\mathrm{G}$ & 243.106 & 1.657 & 245.3 & $\mathrm{~ms}$ \\
$E_{1}-E_{1}$ & 243.076 & 0.417 & & \\
$E_{3}-E_{3}$ & 243.076 & 0.417 & & \\
& & & & & \\
$1 \quad 0 \rightarrow 1$ & 1 & & & & \\
$A_{2}-A_{2}$ & 241.376 & 0.926 & & \\
$\mathrm{G}-\mathrm{G}$ & 241.405 & 1.854 & 239.8 & s \\
$E_{2}-E_{2}$ & 241.4 .33 & 0.464 & & \\
$E_{3}-E_{3}$ & 241.433 & 0.464 & & \\
vs = very strong; s $=$ strong; ms = medium strong
\end{tabular}


Table $6 \mathrm{~b}$. Calculated frequencies (in $\mathrm{cm}^{-1}$ ) and relative intensities for the symmetric $\nu_{24}$ torsion mode (double gearing rotation) in dimethylamine.

\begin{tabular}{|lrrrrr|}
\hline assign. & freq. & int. & exp. ${ }^{a}$ \\
\hline $0 \quad 0 \rightarrow 1$ & 0 & & & & \\
$A_{1}-A_{2}$ & 221.226 & 0.101 & & \\
$\mathrm{G}-\mathrm{G}$ & 221.225 & 0.203 & 219.4 & $\mathrm{~ms}$ \\
$E_{1}-E_{2}$ & 221.224 & 0.051 & & \\
$E_{3}-E_{3}$ & 221.224 & 0.051 & & \\
& & & & \\
$1 \quad 0 \rightarrow 2$ & 0 & & & & \\
$A_{2}-A_{1}$ & 216.386 & 0.023 & & \\
$\mathrm{G}-\mathrm{G}$ & 216.404 & 0.046 & 213.0 & $\mathrm{w}$ \\
$E_{2}-E_{1}$ & 216.423 & 0.012 & & \\
$E_{3}-E_{3}$ & 216.423 & 0.012 & & \\
\hline \multicolumn{5}{r}{${ }^{a} \mathrm{~ms}=$ medium strong; $\mathrm{w}=$ weak. } \\
\hline
\end{tabular}

\subsection{Double Torsion + Bending in Dimethylamine}

Since the introduction of the COC angle bending mode in dimethylether [20] appears to correct the gap between the symmetric and antisymmetric torsion modes, the symmetric bending of the CNC angle was introduced explicitly in the calculations. The non-bonding interactions between the hydrogen atoms during the methyl torsion could be indeed important. The three-dimensional vibration Hamiltonian of dimethylamine may be written as:

$$
\begin{gathered}
H\left(\theta_{1}, \theta_{2}, \beta\right)=-\frac{\partial}{\partial \theta_{1}} B_{11} \frac{\partial}{\partial \theta_{1}}-\frac{\partial}{\partial \theta_{2}} B_{22} \frac{\partial}{\partial \theta_{2}}-\frac{\partial}{\partial \beta} B_{33} \frac{\partial}{\partial \beta} \\
-\frac{\partial}{\partial \theta_{1}} B_{12} \frac{\partial}{\partial \theta_{2}}-\frac{\partial}{\partial \theta_{2}} B_{12} \frac{\partial}{\partial \theta_{1}}-\frac{\partial}{\partial \theta_{1}} B_{13} \frac{\partial}{\partial \beta}-\frac{\partial}{\partial \beta} B_{13} \frac{\partial}{\partial \theta_{1}} \\
-\frac{\partial}{\partial \theta_{2}} B_{23} \frac{\partial}{\partial \beta}-\frac{\partial}{\partial \beta} B_{23} \frac{\partial}{\partial \theta_{2}}+V\left(\theta_{1}, \theta_{2}, \beta\right)
\end{gathered}
$$

where $B_{i j}\left(\theta_{1}, \theta_{2}, \beta\right)$ represents the kinetic energy parameters and $V\left(\theta_{1}, \theta_{2}, \beta\right)$ the three-dimensional potential energy function.

It is easily seen that this three-dimensional Hamiltonian operator is invariant under the same operations that those of the two-dimensional problem, since the bending mode is totally symmetric. The r-NRG for double methyl torsion and bending in dimethylamine will be the $G_{18}$. 
The three-dimensional potential energy function was determined from the electronic energies of 150 selected conformations. Calculations were performed at the RHF/MP2 level with the 6-311G $(\mathrm{d}, \mathrm{p})$ basis set. All the structures were fully optimized taking into account in some way the interactions with the remaining vibration modes.

The same symmetry criterion used before was applied to select the conformations for the torsional coordinates. In addition, five values of the CNC angle around the equilibium, were also selected for describing the variation of the energy with the bending. These angle values were $-5.0^{\circ},-3.0^{\circ} .0 .0^{\circ} 3.0^{\circ}$ and $5.0^{\circ}$. The relative energy values were fitted to the expression for the potential.

In the most stable conformation, the methyl hydrogen atoms do no lie anymore on the CNC plane but stand at $4.0^{\circ}$ and $-4.0^{\circ}$. In the same way, the CNC angle was found to be $111.37^{\circ}$.

The kinetic parameters were determined as before using the Harthcock and Laane procedure $[1,2]$, but now the dependence on the remaining vibrational coordinates was not taken into account because the previous calculations showed that they were very small [18].

The Schrödinger equation was solved by developing the solutions onto 37 $\times 37$ trigonometric functions and 10 harmonic oscillator ones. This basis length implies a Hamiltonian matrix of order 13,690. The symmetry properties of the $\mathrm{G}_{18}$ group permit, however, to factorize this matrix into eight boxes of dimension: $A_{1}(910), A_{2}(780), G(3120), E_{1}(1560), E_{2}(1320)$ and $E_{3}(1440)$. Some of them are degenerate, so that only six matrices have to be diagonalized.

In Table 7 , the energy levels for the double methyl torsion and bending are given. It is seen that as before the levels are clustered into quartets. In the second column of this table, the bending levels are collected. In Table 8 , the frequencies of the torsional bands for the bending fundamental in which the $\mathrm{v} "=0$ are given.

The torsional frequencies obtained in these three-dimensional calculations may be compared with those of the previous example calculated in two dimensions, as well as with the experimental data [19]. It is clear that the introduction of the bending mode displaces the whole spectrum to higher wave-numbers, but modifies the relative position of the symmetric $\nu_{12}$ and antisymmetric $\nu_{24}$ torsion modes. There are no changes in the assignment, but now the gap between the two modes is $37.9 \mathrm{~cm}_{1}$ in better agreement with the experimental value of $36.9 \mathrm{~cm}^{-1}$. The bending levels are found to be too high. 
Table 7. Energy levels (in $\mathrm{cm}^{-1}$ ) for the double methyl torsion and $\mathrm{CNC}$ bending in dimethylamine.

\begin{tabular}{|c|c|c|c|c|c|c|c|c|c|}
\hline$v$ & $v^{\prime}$ & v" & & calc. & $\mathrm{v}$ & $\mathrm{v}^{\prime}$ & $v^{\prime \prime}$ & & calc. \\
\hline \multirow[t]{4}{*}{0} & 0 & 0 & $A_{1}$ & 442.54 & 0 & 0 & 1 & $A_{1}$ & 854.45 \\
\hline & & & $\mathrm{G}$ & 442.40 & & & & $\mathrm{G}$ & 854.45 \\
\hline & & & $E_{1}$ & 442.54 & & & & $E_{1}$ & 854.45 \\
\hline & & & $E_{3}$ & 442.54 & & & & $E_{3}$ & 854.45 \\
\hline \multirow[t]{4}{*}{1} & 0 & 0 & $A_{2}$ & 659.40 & 1 & 0 & 1 & $A_{2}$ & 1071.69 \\
\hline & & & $G$ & 659.40 & & & & $\mathrm{G}$ & 1071.66 \\
\hline & & & $E_{2}$ & 659.40 & & & & $E_{2}$ & 1071.64 \\
\hline & & & $E_{3}$ & 659.40 & & & & $E_{3}$ & 1071.64 \\
\hline \multirow[t]{4}{*}{0} & 1 & 0 & $A_{1}$ & 697.98 & 0 & 1 & 1 & $A_{2}$ & 1108.87 \\
\hline & & & $\mathrm{G}$ & 697.97 & & & & $G$ & 1108.82 \\
\hline & & & $E_{1}$ & 697.97 & & & & $E_{1}$ & 1108.76 \\
\hline & & & $E_{3}$ & 697.97 & & & & $E_{3}$ & 1108.76 \\
\hline \multirow[t]{4}{*}{2} & 0 & 0 & $A_{1}$ & 873.90 & 2 & 0 & 1 & $A_{1}$ & 1280.16 \\
\hline & & & $G$ & 873.91 & 2 & 0 & 1 & $\mathrm{G}$ & 1280.86 \\
\hline & & & $E_{1}$ & 873.93 & & & & $E_{1}$ & 1281.64 \\
\hline & & & $E_{3}$ & 873.93 & & & & $E_{3}$ & 1281.64 \\
\hline \multirow[t]{4}{*}{1} & 1 & 0 & $A_{2}$ & 902.75 & 1 & 1 & 1 & $A_{2}$ & 1321.56 \\
\hline & & & G & 902.77 & & & & G & 1321.83 \\
\hline & & & $E_{2}$ & 902.80 & & & & $E_{2}$ & 132.16 \\
\hline & & & $E_{3}$ & 902.80 & & & & $E_{3}$ & 132.16 \\
\hline \multirow[t]{4}{*}{0} & 2 & 0 & $A_{1}$ & 949.75 & 0 & 2 & 1 & $A_{1}$ & 1361.91 \\
\hline & & & G & 949.76 & & & & $\mathrm{G}$ & 1361.92 \\
\hline & & & $E_{1}$ & 949.77 & & & & $E_{1}$ & 1361.94 \\
\hline & & & $E_{3}$ & 949.77 & & & & $E_{3}$ & 1361.94 \\
\hline \multirow[t]{4}{*}{3} & 0 & 0 & $A_{2}$ & 1085.46 & & & & & \\
\hline & & & $\mathrm{G}$ & 1085.35 & & & & & \\
\hline & & & $E_{2}$ & 1085.24 & & & & & \\
\hline & & & $E_{3}$ & 1085.24 & & & & & \\
\hline \multirow[t]{4}{*}{0} & 3 & 0 & $A_{1}$ & 1196.76 & & & & & \\
\hline & & & $\mathrm{G}$ & 1196.73 & & & & & \\
\hline & & & $E_{1}$ & 1196.69 & & & & & \\
\hline & & & $E_{3}$ & 1196.70 & & & & & \\
\hline
\end{tabular}


Table 8. Frequencies (in $\mathrm{cm}^{-1}$ ) for the symmetric $\nu_{12}$ (antigearing) and antsymmetric $\nu_{24}$ (gearing)torsional modes in dimethylamine.

\begin{tabular}{|c|c|c|c|c|c|c|c|c|c|c|}
\hline $\mathrm{v}$ & $\mathrm{v}^{\prime}$ & $\mathrm{v}^{\prime \prime}$ & & $\mathrm{v}$ & $v^{\prime}$ & $v^{\prime \prime}$ & & calc. & obs.[19] & cal-obs \\
\hline \multicolumn{11}{|c|}{ antigearing mode } \\
\hline \multirow[t]{4}{*}{0} & 0 & 0 & $\rightarrow$ & 0 & 1 & 0 & $A_{1} \rightarrow A_{1}$ & 258.12 & & \\
\hline & & & & & & & $G \rightarrow G$ & 258.12 & 256.3 & 1.8 \\
\hline & & & & & & & $E_{1} \rightarrow E_{1}$ & 258.12 & & \\
\hline & & & & & & & $E_{3} \rightarrow E_{3}$ & 258.12 & & \\
\hline \multirow[t]{4}{*}{0} & 1 & 0 & $\rightarrow$ & 0 & 2 & 0 & $A_{1} \rightarrow A_{1}$ & 254.34 & & \\
\hline & & & & & & & $G \rightarrow G$ & 254.35 & 250.8 & 3.5 \\
\hline & & & & & & & $E_{1} \rightarrow E_{1}$ & 254.36 & & \\
\hline & & & & & & & $E_{3} \rightarrow E_{3}$ & 254.36 & & \\
\hline \multirow[t]{4}{*}{0} & 2 & 0 & $\rightarrow$ & 0 & 3 & 0 & $A_{1} \rightarrow A_{1}$ & 249.46 & & \\
\hline & & & & & & & $G \rightarrow G$ & 249.42 & 245.3 & 4.1 \\
\hline & & & & & & & $E_{1} \rightarrow E_{1}$ & 249.38 & & \\
\hline & & & & & & & $E_{3} \rightarrow E_{3}$ & 249.38 & & \\
\hline \multirow[t]{4}{*}{1} & 0 & 0 & $\rightarrow$ & 1 & 1 & 0 & $A_{2} \rightarrow A_{2}$ & 246.33 & & \\
\hline & & & & & & & $G \rightarrow G$ & 246.35 & 239.8 & 6.5 \\
\hline & & & & & & & $E_{2} \rightarrow E_{2}$ & 246.37 & & \\
\hline & & & & & & & $E_{2} \rightarrow E_{2}$ & 246.37 & & \\
\hline \multicolumn{11}{|c|}{ gearing mode } \\
\hline \multirow[t]{4}{*}{0} & 0 & 0 & $\rightarrow$ & 1 & 0 & 0 & $A_{1} \rightarrow A_{2}$ & 220.18 & & \\
\hline & & & & & & & $G \rightarrow G$ & 220.18 & 219.4 & 0.6 \\
\hline & & & & & & & $E_{1} \rightarrow E_{2}$ & 220.18 & & \\
\hline & & & & & & & $E_{3} \rightarrow E_{3}$ & 220.18 & & \\
\hline \multirow[t]{4}{*}{1} & 0 & 0 & $\rightarrow$ & 2 & 0 & 0 & $A_{2} \rightarrow A_{2}$ & 217.64 & & \\
\hline & & & & & & & $G \rightarrow G$ & 217.65 & 213.0 & 4.6 \\
\hline & & & & & & & $E_{2} \rightarrow E_{1}$ & 217.67 & & \\
\hline & & & & & & & $E_{3} \rightarrow E_{3}$ & 217.67 & & \\
\hline
\end{tabular}




\section{CONCLUSIONS}

The ab initio calculations appear to reproduce relatively well the torsional energy levels as well as the far infrared torsional spectra of methylamine and dimethylamine. The wagging and bending energy levels appear to be too high and the corresponding infrared spectra shift to higher wave-numbers. In addition, the interaction between the wagging and/or bending with the torsion seems to be relatively weak. In the case of methylamine, three-dimensional calculations taking into account simultaneously torsion, wagging and symmetric bending seem to be necessary.

Anyway the present approach appears to be a powerful tool, which could help in the assignment of large-amplitude bands as well as in the prediction of the spectrum.

\section{ACKNOWLEDGEMENTS}

Y.G.S. and M.L.S. wish to thank the "Comision Interministerial de Ciencias y Tecnologia" of Spain for financial support through grant no PB93-0185, M.V. would like to thank the CSIC (Spain) and CONACYT (Mexico) for a sabbatical fellowship. 


\section{References}

[1] D.G. Lister, J.N. MacDonald and N.L.Owen, in "Internal Rotation an Inversion", Academic Press, London, 1978.

[2] H.C. Longuet-Higgins, Mol. Phys.,6, 445 (1963).

[3] Y.G. Smeyers, Adv. Quantum Chem., 24, 1-77, (1992)

[4] Y.G. Smeyers, M.L. Senent, V. Botella and D.C. Moule, J.Chem. Phys., 98, 2754 (1993)

[5] M.A. Harthcock and J. Laane, J. Chem. Phys., 89, 4231 (1985).

[6] Y.G. Smeyers, A. Hernández-Laguna and P.Galera-Gómez, An. Quim. (Madrid), 76, 67 (1980).

[7] J.M. Hollas, in "High Resolution Spectroscopy", Butterworths, London, 1982 .

[8] N. Onashi and J.T. Hougen, J. Mol. Spectrosc., 121, 484 (1987).

[9] Y.G. Smeyers, M. Villa and E. Ortiz, J. Math. Chem., 18, 209-216(1985).

[10] J. Maruani, Y.G. Smeyers and A. Hernández-Laguna, J. Chem. Phys., J. Chem. Phys., 74,3123 (1982).

[11] N. Ohashi, K. Takagi, J.T. Hougen, W.B. Olson and W.J. Lafferty, J. Mol Spectrosc.,126, 443 (1987); ib. 132, 242 (1988).

[12] N. Ohashi, S. Shimada, W.B. Olson, and K. Kawagushi, J. Mol. Spectrosc., 152, 298 (1992).

[13] M. Kreglewski and F. Winther, J. Mol. Spectrosc. 156, 261 (1992).

[14] M. Kreglewski, in "Structure and Conformation of Non-Rigid Molecules", (J. Laane et al Eds.), NATO-ASI Series, pp 29-43, Kluwer, Dordrecht, 1993.

[15] M.J. Firsch, G.W. Trucks, M. Head-Gordon, P.M.W. Gill, M.W. Wong, J.B. Foresman, B.G. Johnson, H. B. Schlegel, M.A. Robb, E.R. Repongle, R. Gomperts, J.L. Andres, K. Raghvachari, J.S. Binkley, C. González, R.L. Martin, D.J. Fox, D,J. Defrees, J. Baker, J.J.P. Stewart and J.A. Pople (Gaussian, Inc. Pittsburg PA, 1992).

[16] Y.G. Smeyers, M. Villa and M.L. Senent, J. Mol. Spectrosc, 177, 66-73 (1996) 
[17] Y.G. Smeyers and A. Niño, J. Comput. Chem.m, 8,380 (1987).

[18] M.L. Senent and Y.G. Smeyers, J. Chem. Phys.,105, 2789 (1996).

[19] J.R. Durig, M.G. Griffin an P. Groner, J. Phys. Chem., 81, 554 (1977).

[20] M.L. Senent, D.C. Moule and Y.G. Smeyers., J. Chem. Phys.,102, 5952 (1995). 